\title{
The five neural pathways of memory
}

\author{
HU Jian-feng \\ Institute of Information Technology, Jiangxi University of Technology, Nanchang 330098, China
}

Keywords: Memory; Neuron; Learning; Brain

\begin{abstract}
All the acquisition of knowledge is a memory, the memory is the foundation of all intellectual activities. Memory is also the basis of education. This paper presents five neural pathways of memory, respectively, based on information, rules, concepts, expectations and integration. These pathways has important significance on the formation and maintain of memory. Understanding these is very helpful to improve education.
\end{abstract}

\section{Introduction}

Memory is the results of modified synaptic transmission. The most prominent bottleneck to understand the mechanisms of memory is the connection structure of fine synapses. However, we still don't know how to connect the brain nerve, how synaptic connection work between what the specific types of neurons, and how synaptic connections modify. The lack of effective research method is important to explore the mechanism of memory, such as brain imaging device resolution and coverage is a contradiction, high resolution is often limited coverage, large coverage often insufficient resolution. Based on combining the various models of learning and memory, combined with some cases of pathological or genius, this paper proposes five neural pathway of memory.

\section{Pathway one: Memory based on information (the basis of information transmission)}

The input data are some sophisticated audio-visual information, following cognitive learning mechanism, then through neural pathway of audio-visual information processing, finally to realize the access of cognitive information by the area of cortex. Most of Williams's syndrome are masters of auditory information memory. A significant feature of these memories is, learning (memory) process is very happy, the performace of children with normal talent is preference for the access to all information of memory by the game way.

A large number of simple information from the outside world enter the brain, including visual, auditory, tactile, olfactory, gustatory, the brain will memory them in simple and selective mode. These memory easily stores but also easy to forget. This channel is the most primitive, but this is the most basic.

\section{Pathway two: Memory based on rule (the basis of habits)}

The input data of allocentric spatial position information is through trial and error learning style or non associative learning way, by the hippocampus and posterior parietal associative or non associative information access. Procedural memory formed in neural network among the motor cortex, striatum, cerebellum and them. Neural correlates of the priming effect is in the neocortex, if you on one occasion unconsciously saw or heard a stimulus, the stimulation appearing again, you will recognize it with 
significantly accelerating speed. The associative learning (classical conditioning and operant conditioning) formed memories stored in the cerebellum, the amygdala and the hippocampus.

This pathway is very important for the habit. Habit can be formed, once formed, are difficult to change. A neural network forming habit is fixed in the brain, do not need too much thought or effort has been completed.

\section{Pathway three: Memory based on concepts (the basis of language)}

Memory access function is realized by the complex contact area by subsystem cognitive learning style. For example, Semantic memory (capital of China is Beijing), Episodic memory (I drink milk in the morning). Declarative memory storage neural network formed among the hippocampus, medial temporal lobe and diencephalon. These structures, including medial temporal lobe neocortex, hippocampus, amygdala and temporal lobe, is vital to declarative memory.

Words and language is not a simple visual and auditory information, it has a specific connotation. If someone did not understand the concept, they can not know the connotation. For example, modern people cannot understand the ancient text, but in ancient times, a child will know. In this pathway, the function of education was began to embody.

\section{Pathway four: Memory based on expected (the basis of grow)}

Memory access function is realized by the complex contact area by subsystem emotional learning style by the limbic system of brain, including the hippocampus, amygdala, cingulate and many other brain structures.

Procrastination: coming from perfectionist's expectations. Too much information, too many decisions, too many choices -- information flooding made us fall into the morass of delay. The studies on physiological research was found that procrastination mostly was around the prefrontal cortex function, where is responsible for the brain's executive function, such as plan, impulse control and attention, also playing the role of filter, droping distracting stimuli from other brain regions. If the prefrontal cortex is damage or low activity, it will lead to a decrease the ability to filter out distracting stimuli, then makes ability of processing task organization becomes poor. In addition to the biological sources, procrastination also includes emotional sources, sense of time deviation and interpersonal origins. In fact procrastinators are most afraid of their own can not fundamentally acceptable. Another cause of procrastination is disputes between of the delay and the time. Procrastinators often look at the time in a mode of "expectation thinking", or they will often take time as a opponent to conquer and win. This attitude toward time aroused more delay.

For some procrastinators, mediocrity is unbearable. They want everything they do very well. These delays can also be called a perfectionist procrastinators. Perfectionists believe that, for a truly great people, things no matter what even more difficult should also be easy. Perfectionist often feel that everything should be to do their own, they believe that any help is a sign of weakness. Even if some help will make things more efficient, perfectionist are also put their's foot down, and they even proud of it. Perfectionists believe that every problem has a correct solution, and find the solution is their duty. Before finding the right solution, they are not willing to take any action, not to undertake any task.

(2) obsessive compulsive disorder: coming from too strict training. Almost all people may have experienced forced phenomenon: for example, sometimes there will be a song sounded in mind frequently, or worry about whether door is not locked after leaving house It can be said that everyone 
may have forced phenomenon. Generally speaking, if this forced phenomenon is slight, short duration, and do not cause serious anxiety disorder, it is a normal performance. Research shows that the neuroendocrine system dysfunction in patients with obsessive compulsive disorder, cause neurotransmitter imbalance, thus the emergence of a variety of obsessive compulsive symptoms.

(3) Seeking advantages and avoiding disadvantages: coming from intuitive. Our brains have a magical ability, which can automaticallydetect errors, and learn from the mistakes of the experience. This kind of mechanism of learning from mistakes is: when the brain once decided to take some action, it has the expected consequences. If the action effect is good, it will release dopamine as a reward reward. If the action effect is bad, or it did not achieve the desired result, it will stop the release of dopamine to punish yourself, let her remember this error, so as to avoid repeating them.

(4) Violence and aggression: coming from extreme reactions. Because these people in emotional fluctuations, are easy to do some harm things to society and themselves. They harm other but get nothing. People all have the desire to conquer. As long as people have the desire to conquer, they will have a tendency to violence, which is to the original human instinct.

\section{Pathway five: Memory based on self (basis of integration)}

The spatial position information based on self-centered input, through a variety of experiential learning or cognitive learning styles, realize memory access through the caudate nucleus to frontal cortex.

This pathway is the most senior type, which will be put with four other pathways together form the deepest memory. This pathway covers most areas of the brain, including the information input, information processing, information storage, information integration. Especially the integration, then is different from the simple memory. Generally speaking, memory is the original reproduction, but in this pathway, in addition to reproduction, more processing is occur. For example, we had experienced the earthquake, this memory is engraved on my heart, but up to now, we might remember not only the earthquake, there may be some dead relatives and friends, small details may be another earthquake, there may be the "pain" of body, there may have a complicated feeling, all these is a simple memory, the brain has already integrated all information.

Parallel processing and serial processing at the same time are the two main coding mode for memory function. Each encoding mode can realize regional encode based on content-addresses and each brain structure and brain cell access data. Also it can realize distributed coding, with less brain cells for more data coding, between the more information to certain brain cells.

\section{Acknowledgements}

This work was supported by Natural Science Foundation of Jiangxi Province [No 20142bab207008], project of Science and Technology Department of Jiangxi Province [No 2013BBE50051] and project of Jiangxi[No. XL1406].

\section{References}

[1] Http://baike.baidu.com

[2] Mihaly Csikszentmihalyi. Finding Flow: The Psychology of Engagement With Everyday Life [M]. $\square$ BasicBooks, 1998. 